\title{
Discovery of the eclipse in the symbiotic binary Z Andromedae
}

\author{
A. Skopal ${ }^{\star}$ \\ Astronomical Institute, Slovak Academy of Sciences, 05960 Tatranská Lomnica, Slovakia \\ Received 10 February 2003 / Accepted 6 March 2003
}

\begin{abstract}
Our photometric observations of the symbiotic binary Z And during its recent (2000-2003) active phase revealed a minimum in the $U, B$ and $V$ light curves (LC) at the position of the inferior conjunction of its cool component (the orbital phase $\varphi=0$ ). This fact and the behaviour of colour indices suggest that the minimum was due to the eclipse of the active hot object by the red giant. Physically plausible fit of the eclipse profile and a precise analysis of the spectral energy distribution (SED) in the ultraviolet continuum suggest a disk-like structure for the hot object during active phases. The present knowledge of fundamental parameters of the system limits the orbital inclination $i$ to $76^{\circ}-90^{\circ}$. The presence of the Rayleigh attenuated far-UV continuum at $\varphi \sim 0$ during quiescent phase confirms the very high inclination of the $\mathrm{Z}$ And orbit.
\end{abstract}

Key words. stars: binaries: symbiotics - stars: individual: Z And

\section{Introduction}

$\mathrm{Z}$ And is the prototype of the symbiotic stars. Its brightness variation has been recorded from 1887 (e.g. Mattei 1978, and reference therein). The historical LC shows several active phases during which fluctuations ranges in amplitude from a few tenths of a magnitude to about 3 magnitudes (e.g. Formiggini \& Leibowitz 1994). During active phases the hot component expands in radius and becomes significantly cooler. A mass-outflow from the active star is indicated directly by a broadening of emission lines and/or by profiles of the P-Cygni type (e.g. Fernández-Castro et al. 1995, hereafter FC95; Swings \& Struve 1941). The quiescent phase of Z And is characterized by a complex wave-like brightness variation as a function of the orbital phase (e.g. Skopal 2001a). Based on all available primary minima in the LC from 1900 to 1996, Skopal (1998) determined their ephemeris as

$\mathrm{JD}_{\text {Min }}=2414625.2+757.5( \pm 0.5) \times E$,

which is identical (within uncertainties) with timing of the inferior spectroscopic conjunction of the giant star in the system (Mikolajewska \& Kenyon 1996, hereafter MK96). We use this ephemeris further in this letter.

The binary of Z And composes of a late-type, M4.5 III, giant (Mürset \& Schmid 1999) with $M_{\mathrm{g}} \sim 2 M_{\odot}, R_{\mathrm{g}} \sim 85-140 R_{\odot}$ and $L_{\mathrm{g}} \sim 880 L_{\odot}$ (Nussbaumer \& Vogel 1989, hereafter NV89; MK96; Tomov et al. 2003). The hot component is probably a magnetic accreting white dwarf (Sokoloski \& Bildsten 1999) with $M_{\mathrm{h}} \sim 0.5-1 M_{\odot}, L_{\mathrm{h}} \approx 900-2500 L_{\odot}$, and $T_{\mathrm{eff}} \sim 10^{5} \mathrm{~K}$, surrounded by an ionized nebula (MK96; FC95).

$\mathrm{Z}$ And is considered to be a non-eclipsing binary. MK96 suggested the orbital inclination $i \approx 50^{\circ}-70^{\circ}$ as a compromise

* e-mail: skopal@ta3.sk between the behaviour of the He II 1640 emission - showing a deep minimum around the giant's inferior conjunction - and the lack of eclipses in the LC. Also Schmid \& Schild (1997), based on repeated polarimetric measurements, determined the orbital inclination to $47^{\circ} \pm 12^{\circ}$.

In this letter we report the first detection of the eclipse in the $\mathrm{LC}$, which provides direct evidence for a very high inclination of the orbital plane of $\mathrm{Z}$ And.

\section{Observations}

Our photometric $U, B, V, R$ measurements of $\mathrm{Z}$ And were performed in the standard Johnson system using single-channel photoelectric photometers mounted in the Cassegrain foci of 0.6-m reflectors at the Skalnaté Pleso and Stará Lesná observatories. $\mathrm{Z}$ And was measured with respect to the comparison star SAO $53150(V=8.99, B-V=0.41, U-B=0.14$, $V-R=0.16$ ) which was checked by the previous standard star SAO $35642(V=5.30, B-V=-0.06, U-B=-0.15$, $V-R=-0.04)$. To cover gaps in photoelectric observations we also used visual magnitude estimates gathered by the members of Association Française des Observateurs d'Étoiles Variables (AFOEV), which are available from the CDS. In addition, we compared a few V-CCD observations, which are available from the VSNET database. Figure 1 shows the LCs covering the recent active phase of $Z$ And. A table containing the data till 2001 December and other details about photoelectric observations can be found in Skopal et al. (2002).

Finally, we also used the low resolution spectra available from the final IUE archive (see Sect. 3.3). They were taken on 06/04/84 (SWP22684 + LWP03099), 24/12/85 (SWP27370 + LWP07370), 11/07/86 (SWP28655 + LWP08585) and 03/02/88 (SWP32845 + LWP12614). We dereddened the 


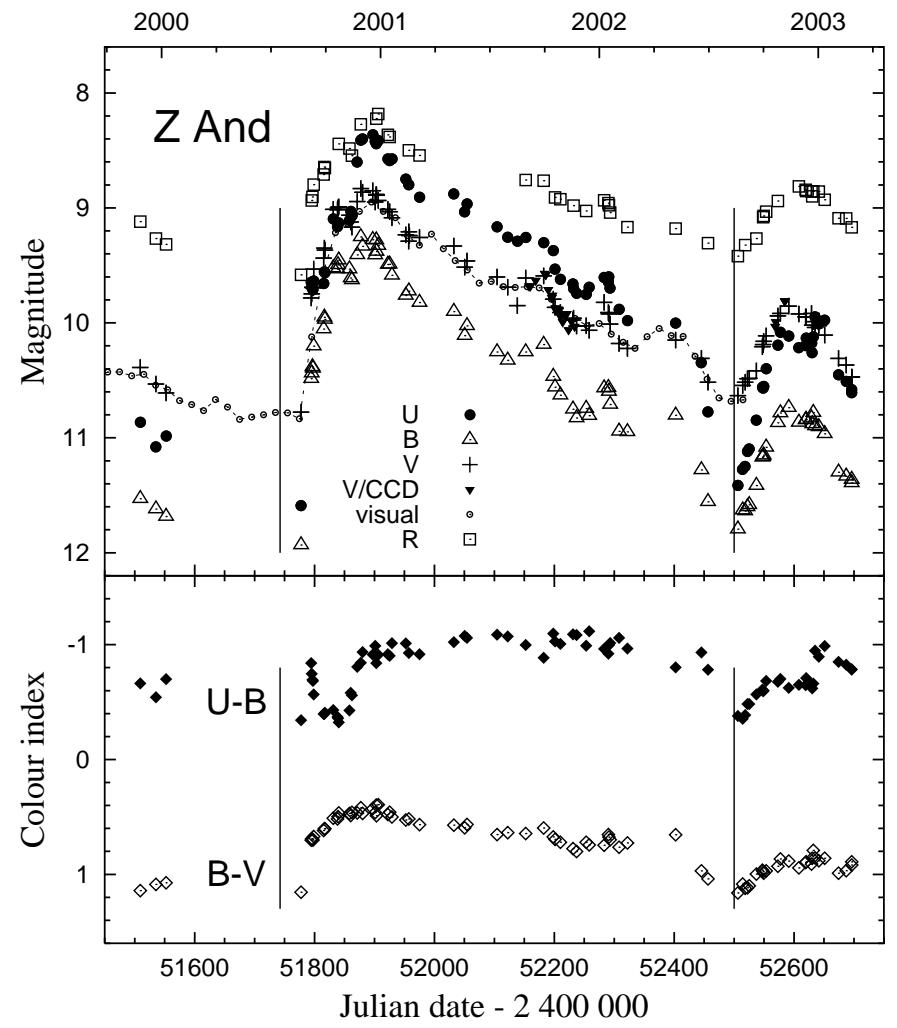

Fig. 1. The $U B V R$ photometry of $\mathrm{Z}$ And covering the current active phase. The vertical lines denote the position of the inferior spectroscopic conjunction of the giant according to the ephemeris given by Eq. (1). Small circles connected with a broken line represent 20-day means of the visual estimates from CDS.

spectra with $E_{B-V}=0.30$ (NV89) by using the extinction curve of Cardelli et al. (1989).

\section{Results}

\subsection{Evidence of the eclipse}

Figure 1 shows the $U B V R$ LCs of $\mathrm{Z}$ And covering its current outburst, which started in 2000 September (Skopal et al. 2000). The star's brightness reached a maximum at the beginning of 2000 December, after which it has been gradually decreasing. The latest observations from the end of 2002 showed a transient small increase of the activity. The most pronounced feature in the LC is a deep minimum, which occurred around JD 2452 500. We summarize its basic observational characteristics as follows:

1. Position of the minimum agrees perfectly with the time of the inferior spectroscopic conjunction of the cool component in the binary (cf. Eq. (1)).

2. At the mid of the minimum (JD 2452500 ), Z And became considerably redder than before/after its beginning/end: The colour indices were identical with those observed prior to the outburst (cf. bottom panel of Fig. 1) and its brightness was very close to these values.

These facts imply that the minimum was due to the eclipse of the active hot star by the red giant.

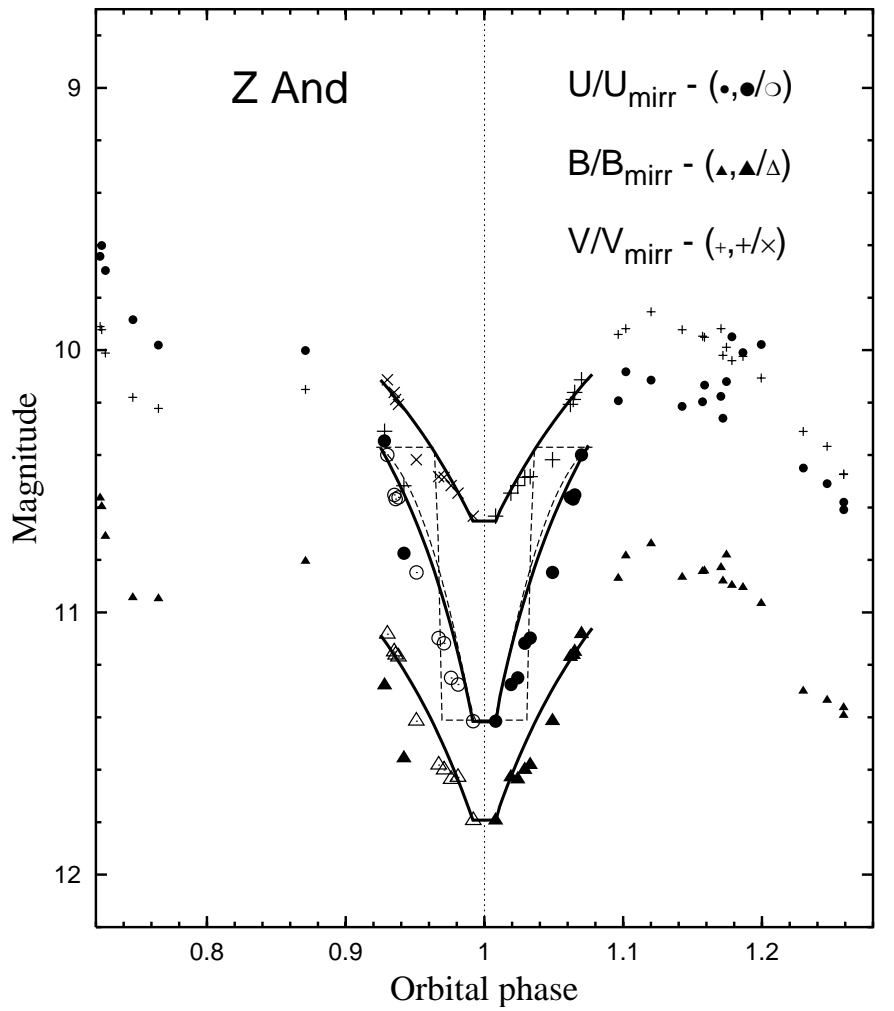

Fig. 2. Comparison of the observed and the modeled eclipse profile. Solid thick line corresponds to the model of an extended belt, which is subject to the eclipse. This model suggests a disk-like structure of the hot active object and limits the orbital inclination to $76^{\circ}-90^{\circ}$. Models with a spherical shape of the hot component (dashed lines) do not match observations (the rectangular shape) or are not physically plausible (the broad dashed profile). See Sect. 3.2 for more details.

\subsection{Geometry of the eclipsed object}

The observed eclipse profile is basically of a V-type. However, its shape cannot be accurately determined, because of (i) a poor coverage of the descending branch by observations, (ii) an influence of a strong nebular contribution, which makes the top parts of the profile broader, and (iii) an enhanced activity during the time when the hot object was arising up from the eclipse. Second point is given by a strong nebular contribution in the spectrum during active phases (Sect. 3.3), and the third point is indicated by a higher level of the star's brightness after the eclipse then prior to it, although a reverse behaviour was expected due to a general decrease from the major outburst. The basic V-type profile of the eclipse and its position are also supported by continuous visual observations from CDS (Fig. 1).

Therefore, to compare better the observed profile to a model, we mirrored the data from the ascending branch of the eclipse with respect to the time of the spectroscopic conjunction at the epoch $E=50$ (JD 2452500.2 , Eq. (1)). Figure 2 shows the result, which suggests that radii of both the eclipsing and the eclipsed object are relatively large and comparable each to other. Based on fundamental parameters published by NV89, MK96, and Tomov et al. (2003), we derived the range of the giant's radius as $R_{\mathrm{g}} \sim(0.17-0.32) A$ and limited the size of 
the eclipsed object by its Roche lobe radius, $R_{\text {ecl }}^{\mathrm{L}}$, as $R_{\text {ecl }}<$ $R_{\text {ecl }}^{\mathrm{L}} \sim(0.26-0.32) A$ ( $A$ stands for the separation between the centers of mass of the binary components). First we tried to match the eclipse profile for a spherical shape of the hot object by using a code of Kvačkay (1994). However, its luminosity and temperature during active phases, $L_{\mathrm{h}} \approx 2500-3600 L_{\odot}$ and $T_{\mathrm{h}}=5-3 \times 10^{4} \mathrm{~K}$ (FC95; Tomov et al. 2003), imply an effective radius only of $R_{\mathrm{ecl}} \approx 2 R_{\odot}$, which cannot reproduce the observed eclipse profile (Fig. 2). Contrary, a large sphere $\left(R_{\mathrm{ecl}} \approx 100 R_{\odot}\right)$, which can reproduce formally the eclipse profile, has an unrealistically high luminosity $\left(L_{\mathrm{h}} \approx 10^{5} L_{\odot}\right)$. Therefore, we rejected models with the spherical shape of the eclipsed object. Second, we matched the observed profile by a simple model of a uniformly radiating belt around the central star as the eclipsed object by using own code. Such the model can simulate, for example, the geometry of an accretion disk seen edge-on, which is extended at its outer parts. Here we obtained the following solutions:

(i) If the linear size of the giant's stellar disk that eclipses the belt, $R_{\mathrm{g}}^{\mathrm{E}}$, is larger than $R_{\mathrm{ecl}}$, the resulting models are determined by $R_{\text {ecl }} / A=0.21, R_{\mathrm{g}} / A=0.26\left(i=90^{\circ}\right)-0.32\left(i=79^{\circ}\right)$ and the belt radiates $63 \%, 49 \%$ and $40 \%$ of the total light in the $U, B$ and $V$ band, respectively.

(ii) For $R_{\mathrm{g}}^{\mathrm{E}}<R_{\mathrm{ecl}}$, the models correspond to $R_{\mathrm{ecl}} / A=0.26$, $R_{\mathrm{g}} / A=0.21\left(i=90^{\circ}\right)-0.32\left(i=76^{\circ}\right)$ and the belt contributes $78 \%, 61 \%$ and $50 \%$ of the total light in the $U, B$ and $V$ band, respectively. Figure 2 shows example given by parameters $R_{\mathrm{g}} / A=R_{\mathrm{ecl}} / A=0.26$ and $i=81^{\circ}$ of the case (ii).

Analysis of this section suggests that the hot eclipsed object during the activity has a disk-like structure. This result is supported independently by our analysis of the SED, which we present in the following section.

\subsection{SED in the ultraviolet continuum}

The effect of eclipses of a multi-component source of radiation depends on a physical displacement and radiative contributions of its individual components in the system. In the case that a significant fraction of radiation at the wavelength under consideration comes from the region, which is subject to eclipse, a minimum in the LC is well observable. In the contrary case, the eclipse effect is very faint. In addition, the displacement and relative contributions of emission regions in a symbiotic system are a strong function of the level of the activity. Therefore, to demonstrate this situation for $\mathrm{Z}$ And, we use our method of a precise modeling of its ultraviolet continuum during both the active and the quiescent phase.

In our procedure we assume that the observed flux, $F_{\lambda}\left[\mathrm{erg} \mathrm{cm}^{-2} \mathrm{~s}^{-1} \AA^{-1}\right]$, is given by superposition of fluxes from a hot stellar object and a nebula. We approximate the former by blackbody radiation, which can be Rayleigh attenuated, and for the latter we assume the f-b plus f-f transitions in fully ionized hydrogen and helium. So, we express the observed flux as

$F_{\lambda}=k_{\mathrm{h}} \times \pi B_{\lambda}\left(T_{\mathrm{h}}\right) \mathrm{e}^{-n_{\mathrm{H}} \sigma_{\lambda}^{\mathrm{R}}}+k_{\mathrm{N}} \times \varepsilon_{\lambda}\left(T_{\mathrm{e}}, a\right)$.

The first term represents radiation of the blackbody at a temperature $T_{\mathrm{h}}$, which is attenuated by neutral atoms of hydrogen on the line of sight due to the Rayleigh scattering process
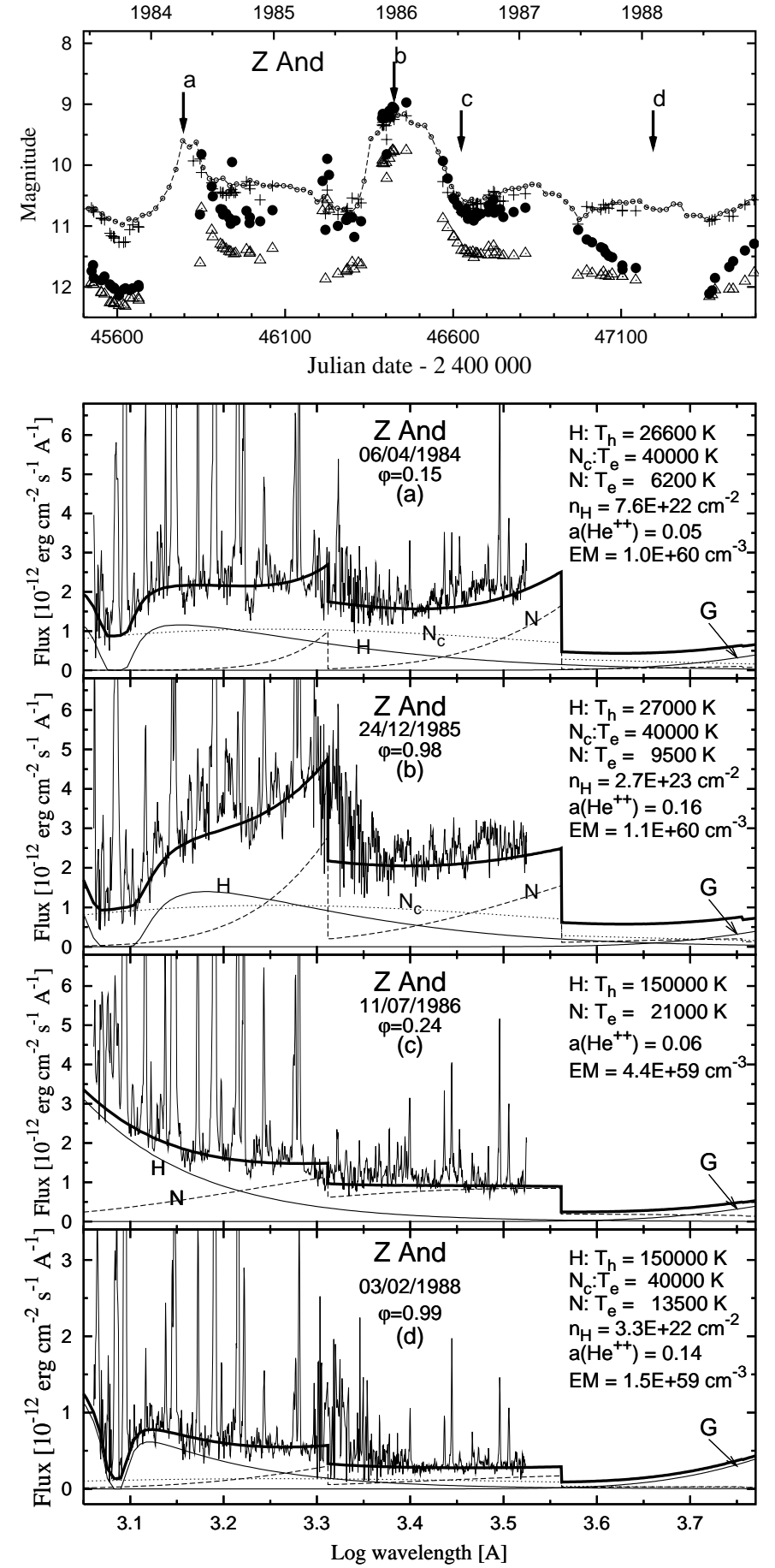

Fig. 3. Reconstructed SED in the UV continuum of Z And during its active and quiescent phases (panels a), b) and c), d), respectively). Solid thin lines $(\mathrm{H}, \mathrm{G})$ represent stellar components of radiation from the hot object and the red giant $\left(T_{\mathrm{g}}=2800 \mathrm{~K}\right)$. Dashed and dotted lines $\left(\mathrm{N}, \mathrm{N}_{\mathrm{c}}\right)$ represent the nebular components of radiation and the solid thick line is the resulting modeled continuum (Sect. 3.3). Corresponding parameters are displayed at the top-right corner. Emission measure was calculated for $d=1.12 \mathrm{kpc}$. Denotation of LCs in the top panel is the same as in Fig. 1. Double component temperature spectrum, which develops during the activity (panels a) and b)), suggests a disk-like structure of the hot object, which is seen approximately edge-on. The Rayleigh attenuated far-UV continuum, seen even during quiescence at $\varphi \sim 0$, confirms the high inclination of the orbital plane (panel d)). 
$\left(n_{\mathrm{H}}\left[\mathrm{cm}^{-2}\right]\right.$ is the column density of $\mathrm{H}$ atoms, $\sigma_{\lambda}^{\mathrm{R}}\left[\mathrm{cm}^{2}\right]$ is the Rayleigh scattering cross-section for atomic hydrogen and $k_{\mathrm{h}}$ is a dimensionless scaling factor). The second term represents the nebular radiation given by the volume emission coefficient,

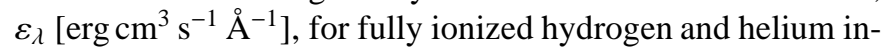
cluding all acts of recombination (f-b transitions) and thermal bremsstrahlung (f- $f$ transitions). The coefficient is scaled to the observed nebular flux with the factor $k_{\mathrm{N}}=E M / 4 \pi d^{2}\left[\mathrm{~cm}^{-5}\right]$ ( $E M$ is the emission measure and $d$ is the distance to the object) and was calculated by the same way as used by Skopal (2001b). Finally, $a=n\left(\mathrm{He}^{++}\right) / n(\mathrm{H})$ is the abundance of doubly ionized helium and $T_{\mathrm{e}}$ is electron temperature.

First, we determined fluxes in the continuum at selected wavelengths. Because of noise, numerous emission lines and absorption features we preferred an estimate by eye. Second, we calculated a grid of models given by Eq. (2) for 6 parameters, $k_{\mathrm{h}}, T_{\mathrm{h}}, n_{\mathrm{H}}, k_{\mathrm{N}}, T_{\mathrm{e}}, a$, and selected that giving the best fit to the observed fluxes under conditions of the least square method. Figure 3 shows a comparison of the observed spectra and the resulting models during active and quiescent phases. The resulting UV continuum from active phases (panels a), b) of Fig. 3) consists of two components of radiation of a very different temperature regimes: a relatively cool stellar component $\left(T_{\mathrm{h}} \sim 27000 \mathrm{~K}\right)$, which is Rayleigh attenuated at the far$\mathrm{UV}$, and a very hot radiation, which gives rise to highly ionized emission lines and the nebular continuum. The latter consists of two - low plus high temperature - components. The hot nebula had to be added to fit the far-UV continuum. With analogy to AR Pav, the electron temperature of $40000 \mathrm{~K}$ was adopted here (cf. Skopal 2003). In the quiescent phase (panels c), d) of Fig. 3), the stellar component of radiation corresponds to very high temperature $T_{\mathrm{h}} \sim 150000 \mathrm{~K}$ (NV89), the low temperature nebular emission dominates the near-UV region and the hot coronal emission is very faint. Around the inferior conjunction of the giant, the far-UV continuum is Rayleigh attenuated. Below we summarize results of this analysis, which confirm those obtained by modeling the eclipse profile:

(i) Double component temperature spectrum with the Rayleigh attenuated stellar component of radiation, which develops during active phases, suggests a disk-like structure for the eclipsed object seen approximately edge-on. Such configuration implies that the radiation from the hot central star $\left(T_{\mathrm{h}} \sim 150000 \mathrm{~K}\right)$ is absorbed and diffused in the disk in the direction to the observer, and thus transformed to the observed $T_{\mathrm{h}} \sim 27000 \mathrm{~K}$, but it is free in directions to the poles giving rise to the nebular emission there (panels a), b) of Fig. 3).

(ii) The presence of the Rayleigh attenuated far-UV continuum at $\varphi \sim 0$ even during the quiescent phase is a consequence of the high orbital inclination - a typical feature for eclipsing symbiotic binaries during quiescent phases (Isliker et al. 1989). In this case the hot stellar radiation is scattered on the neutral atoms of hydrogen in the giant's wind at positions around $\varphi \sim 0$, but it is not present at $\varphi \gtrsim 0.1$ (panels c), d) of Fig. 3).

(iii) At the maximum and during quiescence the nebular emission dominates the optical, which means that a significant fraction of the total hot object radiation can be physically displaced from the central hot star. By other words, a fraction of the radiation removed from the line of sight due to the eclipse can be very small, which precludes arising the eclipse effect in the LC. The eclipse effect can be observed only at specific brightness phases, at which the radiative contribution from a pseudophotosphere in the optical rivals that from the nebula.

\section{Conclusions}

The results of this letter can be summarized as follows:

(i) Our photometry of Z And during its recent active phase (2000-2003) revealed a deep minimum in the $U, B$ and $V$ LCs.

(ii) The position of the minimum at the inferior conjunction of the cool component in the binary and the behaviour of colour indices strongly suggest that the minimum was caused by the eclipse of the active hot object by the red giant.

(iii) Physically plausible fit of the eclipse profile suggests a disk-like structure for the active hot object. Our analysis of the SED in the UV continuum during the previous (1984-1986) active phase confirmed this result. The present knowledge of fundamental parameters of the system restricts the orbital inclination to $76^{\circ}-90^{\circ}$.

(iv) The presence of the Rayleigh attenuated far-UV continuum even during the quiescent phase at $\varphi \sim 0$ confirms the very high inclination of the $\mathrm{Z}$ And orbit.

(v) A dominance of the nebular component of radiation in the optical during the maximum and quiescence makes it difficult to observe the eclipse effect. It can be observed only at specific brightness phases.

Acknowledgements. This work used in part the visual estimates of $\mathrm{Z}$ And gathered by the members of AFOEV available from the CDS database and CCD observations made by Makoto Watanabe and Dong West available from the VSNET database. This research has been supported by the Slovak Academy of Science under a grant No. 1157.

\section{References}

Cardelli, J. A., Clayton, G. C., \& Mathis, J. S. 1989, ApJ, 345, 245

Fernández-Castro, T., González-Riestra, R., Cassatella, A., Taylor, A. R., \& Seaquist, E. R. 1995, ApJ, 442, 366 (FC95)

Formiggini, L., \& Leibowitz, E. M. 1994, A\&A, 292, 534

Isliker, H., Nussbaumer, H., \& Vogel, M. 1989, A\&A, 219, 271

Kvačkay, P. 1994, private communication

Mattei, J. A. 1978, J. Roy. Astron. Soc. Can., 72, 61

Mürset, U., \& Schmid, H. M. 1999, A\&AS, 137, 473

Mikolajewska, J., \& Kenyon, S. J. 1996, AJ, 112, 1659 (MK96)

Nussbaumer, H., \& Vogel, M. 1989, A\&A, 213, 137 (NV89)

Schmid, H. M., \& Schild, H. 1997, A\&A, 327, 219

Skopal, A. 1998, A\&A, 338, 599

Skopal, A. 2001a, A\&A, 366, 157

Skopal, A. 2001b, Contrib. Obs. Skalnate Pleso, 31, 119

Skopal, A. 2003, New Astron., in press [astro-ph/0302542]

Skopal, A., Chochol, D., Pribulla, T., \& Vaňko, M. 2000, IBVS No. 5005

Skopal, A., Vaňko, M., Pribulla, T., et al. 2002, Contrib. Obs. Skalnate Pleso, 32, 62

Sokoloski, J. L., \& Bildsten, L. 1999, ApJ, 517, 919

Swings, P., \& Struve, O. 1941, ApJ, 93, 356

Tomov, N. A., Taranova, O., \& Tomova, M. 2003, A\&A, in press 